\title{
Percepção e Uso de Tecnologias da Informação e Comunicação por Psicoterapeutas
}

\author{
Sílvia Cristina Marceliano Hallberg \\ Carolina Saraiva de Macedo Lisboa ${ }^{1}$ \\ Programa de Pós-graduação em Psicologia da Pontifícia Universidade Católica \\ do Rio Grande do Sul, Porto Alegre, RS, Brasil
}

\begin{abstract}
Resumo
O presente trabalho relata e discute os resultados de uma pesquisa que objetivou investigar a percepção e o uso de tecnologias da informação e comunicação (TICs) por psicoterapeutas. O delineamento do estudo foi quantitativo, exploratório, descritivo e de corte transversal com amostra de 155 psicoterapeutas, graduados em psicologia, entre 23 e 72 anos $(M=36,74 ; D P=11,45)$. Esses clínicos responderam a um questionário online sobre percepção e uso de TICs. Os resultados apontam que as TICs estão significativamente presentes na vida dos psicoterapeutas, mas no âmbito profissional seu uso é mais restrito. Com exceção de pedidos de solicitação de amizades de pacientes, via Facebook, os clínicos pesquisados não se sentem desconfortáveis ao se comunicarem com pacientes através de TICs. Os participantes se mostraram indecisos quanto às percepções a respeito das resoluções legais sobre TICs e psicoterapia. Alerta-se para a necessidade de mais estudos empíricos sobre uso de redes sociais por psicoterapeutas. Sugere-se o desenvolvimento de estudos randomizados, com amostra nacional, para uma descrição mais generalizada acerca do uso de TICs por psicoterapeutas brasileiros, assim como estudos de delineamento qualitativo para aprofundamento da compreensão desse tema.
\end{abstract}

Palavras-chave: Psicoterapia, computadores, internet, telefone celular, redes sociais.

\section{Information and Communications Technologies' Perception and Use by Psychotherapists}

\begin{abstract}
This paper reports and discusses the results of a study that investigated the perception and the use of information and communication technologies (ICTs) by psychotherapists. The design of the study was quantitative, exploratory, descriptive and cross-sectional with a sample of 155 psychotherapists, graduated in psychology, between 23 and 72 years $(M=36.74, S D=11.45)$. These clinical professionals answered to an online questionnaire about perception and use of ICTs. The results showed that ICTs are significantly present in the daily lives of psychotherapists, but their use in the professional context is more restricted. Except regarding the request of friendships, via Facebook by patients, the clinical professionals do not feel uncomfortable in communicating with patients through ICT. The participants were in doubt about their perceptions of the legal resolutions on ICT and psychotherapy. It was evidenced the need for more empirical studies about the use of social networks by psychotherapists.
\end{abstract}

Endereço para correspondência: Pontifícia Universidade Católica do Rio Grande do Sul, Programa de PósGraduação em Psicologia, Av. Ipiranga, 6681, Prédio 11, Sala 929, Partenon, Porto Alegre, RS, Brasil 90619900. Fone: +55 (51) 3320.7739. E-mail: carolina.lisboa@pucrs.br 
The development of studies with a randomized national sample for a more general description about the use of ICT by Brazilian psychotherapists, as well as qualitative design studies to reach a better understanding of this subject are suggested.

Keywords: Psychotherapy, computers, internet, mobile phone, social networking.

\section{Percepción y Uso de las Tecnologías de la Información y Comunicación por Psicoterapeutas}

\section{Resumen}

Este trabajo describe y analiza los resultados de un estudio que investigó la percepción y el uso de tecnologías de la información y la comunicación (TIC) por psicoterapeutas. El diseño fue cuantitativo, exploratorio, descriptivo y transversal con una muestra de 155 psicoterapeutas, licenciados en psicología, entre 23 y 72 años $(M=36.74, S D=11.45)$. Estos respondieron a un cuestionario en línea acerca de la percepción y el uso de las TIC. Los resultados muestran que las TIC son significativamente presente en las vidas de los psicoterapeutas, sino en el uso profesional es más restringido. Excepto con la solicitud de amistad de los pacientes en Facebook, los psicoterapeutas no se sienten incómodos cuando se comunican con los pacientes a través de las TIC. Los participantes estaban indecisos en cuanto a las percepciones acerca de las resoluciones judiciales sobre las TIC y la psicoterapia. Alertase para la necesidad de más estudios empíricos sobre el uso de las redes sociales por psicoterapeutas. Se sugere el desarollo de estudios aleatorizados con una muestra nacional, para obtener una descripción más general sobre el uso de las TIC, así como los estudios de diseño cualitativos para desarrollar una mejor comprensión de este tema.

Palabras clave: Psicoterapia, ordenadores, internet, teléfono móvil, red social.

As tecnologias da informação e comunicação (TICs) representam um conjunto de dispositivos, serviços e conhecimentos relacionados a uma determinada infraestrutura, composta por computadores, softwares e sistemas de redes, os quais possuem a capacidade de reproduzir, processar e distribuir informações para pessoas e organizações. São exemplos de TICs os computadores pessoais, telefones celulares, a internet, o correio eletrônico, os suportes de armazenamentos de dados, a TV digital e as diversas tecnologias digitais de acesso remoto e de captura e tratamento de dados, sejam eles textos, imagens ou som (Veloso, 2011). As TICs estão cada vez mais presentes no cotidiano dos brasileiros. De acordo com os recentes dados do Instituto Brasileiro de Geografia e Estatística - IBGE (2011) 77,7 milhões de pessoas de 10 anos ou mais de idade acessam a $w e b$, o que equivale a $46,5 \%$ do total da população. De 2005 para 2011, houve um aumento de $143,8 \%$ do contingente de inter- nautas no país e $66 \%$ desses utilizam a rede diariamente. O crescimento do número de brasileiros que possuem telefone celular foi de 107,2\% (Comitê Gestor da Internet no Brasil [CGI], 2012; IBGE, 2011).

A psicoterapia corresponde a uma técnica de compreensão, análise e intervenção própria do psicólogo, que se realiza através da aplicação sistematizada e controlada de métodos e técnicas psicológicas, reconhecidos pela ciência, pela prática e pela ética profissional. Essa técnica promove a saúde mental e propicia condições para o enfrentamento de conflitos e/ou transtornos psíquicos de indivíduos ou grupos (Conselho Federal de Psicologia [CFP], 2000). Os recentes avanços tecnológicos têm impactado significativamente a prestação de psicoterapia e outros serviços clínicos (Barnett, 2011). A psicoterapia tem sido influenciada pelas TICs, pela cultura digital e mídias sociais (Kolmes, 2012). O psicoterapeuta, na atualidade, depara-se com 
a presença dessas novas tecnologias seja através do impacto que essas têm provocado na subjetividade de pacientes e terapeutas, através da criação de novas modalidades de atendimento, como as online, ou através das novas possibilidades de contato à distância com pacientes como as redes sociais (Abreu, Leitão, \& Nicolaci-da-Costa, 2005; Levisky \& Silva, 2010; Meyer \& Prado, 2006; Sfoggia et al., 2014). A velocidade com que essas tecnologias vêm permeando o mundo tem gerado uma proliferação de novas oportunidades, questões e desafios para o psicoterapeuta (Barnett, 2011; Kolmes, 2012; McMinn, Bearse, Heyne, Smithberger, \& Erb, 2011). Desse modo, a maioria dos clínicos, na atualidade, parece experimentar dúvidas quanto à utilização mais eticamente adequada de vários tipos de tecnologias em seus cotidianos profissionais (McMinn et al., 2011). Embora exista uma tendência de crescimento do papel dessas novas tecnologias da informação e comunicação no campo das psicoterapias (Silverman, 2013), o manejo da internet (Leitão, 2003) e de outras formas de TICs (McMinn et al., 2011) por psicoterapeutas em suas práticas clínicas ainda se mostra restrito. Em uma consulta a 296 psicólogos norte-americanos sobre esse tema, localizou-se uma taxa relativamente baixa de uso de TICs, além de elevada preocupação ética quanto à utilização dessas ferramentas no campo profissional, especialmente quanto ao manejo de sites de redes sociais. As ações em tecnologia mais frequentemente praticadas por esses clínicos envolviam armazenar registros de psicoterapia em um computador, com proteção de senha; corresponder-se eletronicamente com companhias de seguros de saúde e passar informações confidenciais de um paciente, via fax, para outro prestador de cuidados de saúde. A maioria desses profissionais investigados $(79,3 \%)$ nunca divulgou seus serviços na internet. Apenas $0,7 \%$ desses psicólogos declarou prestar serviços clínicos via Skype frequentemente. Somente 5,5\% afirmou executar frequentemente serviços por email e $12,5 \%$ se comunicam com pacientes para agendamento de consultas clínicas por essa via. Serviços psicológicos via telefone celular eram frequentemente realizados por $11,25 \%$ dos consultados e somen- te $1,4 \%$ desses serviços eram prestados através de trocas de mensagens de texto do tipo SMS/ torpedo. A maioria desses psicólogos consultados acredita não ser ético prestar serviços clínicos por meio de um site de rede social $(80,3 \%)$ e $71,5 \%$ não possui um perfil pessoal no Facebook. A grande maioria nunca permitiu que um cliente tivesse acesso completo $(96,9 \%)$ ou mesmo acesso limitado $(95,8 \%)$ a sua página pessoal no Facebook (McMinn et al., 2011).

Não existem estudos empíricos, atuais e com amostras nacionais, que descrevam a forma como psicoterapeutas têm percebido e lidado com as TICs em seus cotidianos pessoais e profissionais. Um estudo mais recente sobre essa temática data do começo dos anos 2000, quase uma década após a popularização da internet e dos aparelhos de telefone celular no Brasil (Carvalho, 2006; Leitão \& Nicolaci-da-Costa, 2001). $\mathrm{O}$ referido estudo apresentava uma investigação qualitativa com 16 psicoterapeutas brasileiros a respeito dos impactos da internet na realidade de seus consultórios. Constatou-se que, apesar de perceberem a internet cada vez mais presente na vida de seus atendidos, os psicoterapeutas faziam pouco uso pessoal e profissional dessa tecnologia (Leitão, 2003).

Revelou-se ainda que os psicoterapeutas sentiam-se confusos, desorientados e inseguros em relação à entrada da internet em seus consultórios e práticas clínicas (Abreu et al., 2005; Leitão, 2003). Todos os 16 psicoterapeutas entrevistados eram usuários da internet, mas, em sua maioria, utilizavam-na relativamente pouco e para objetivos muito específicos. O manejo da web estava mais vinculado à troca emails e realização de pesquisas relacionadas a tarefas laborais. Para esses clínicos a rede era percebida como um instrumento de trabalho e um suporte para suas práticas profissionais. Seu uso pessoal, fora do contexto clínico, foi descrito como moderado e controlado, inclusive os entrevistados declararam colocar limites claros e precisos para o uso da rede. Conhecer pessoas via rede, bater papo, jogar ou realizar outras atividades pessoais e de lazer dificilmente faziam parte dos hábitos desses psicoterapeutas. Poucos foram os clínicos que declararam utilizar qualquer forma de co- 
municação online com seus pacientes. Esses psicoterapeutas acessavam a rede com frequência bastante irregular e poucos se conectavam diariamente. Esses terapeutas também afirmaram não encontrar atrativos na internet. A rede não despertava curiosidade ou diversão e funcionava somente como uma ferramenta de trabalho útil. Somente 4 dos 16 entrevistados referiu trocar emails sobre assuntos pessoais. Além de usarem o correio eletrônico, alguns psicoterapeutas realizavam consultas esporádicas em sites de psicologia. Em sua maioria, limitavam suas atividades na web para troca de emails de cunho profissional (Leitão, 2003).

Somada a escassez de produções empíricas com amostras nacionais e recentes sobre a percepção e manejo de TICs por psicoterapeutas, há uma carência de determinações legais mais específicas sobre a temática dos atendimentos psicoterápicos e uso de tecnologias. Não há, no país, determinações que regulem ou orientem psicólogos quanto à condução de contatos com pacientes via email, mensagens instantâneas através do celular, ferramentas de videoconferência, como o Skype, redes sociais, como o Facebook. Nem mesmo o mais recente pronunciamento do CFP (2012) a respeito de serviços psicológicos realizados por meios tecnológicos de comunicação à distância (Resolução CFP $n^{\circ}$ 11/2012) contempla essa temática. Essa mesma lacuna pode ser encontrada no vigente Código de Ética Profissional do Psicólogo (CFP, 2005). Os pronunciamentos legais sobre uso de tecnologias por psicoterapeutas existentes, no país, tratam somente da prestação de serviços online. O Conselho Federal de Medicina - CFM manifesta-se proibindo consultas médicas virtuais para substituir atendimentos presenciais de pacientes (CFM, 2011). O Conselho Federal de Psicologia - CFP regulamenta que as psicoterapias conduzidas por meio de recursos tecnológicos de comunicação à distância são de caráter exclusivamente experimental no Brasil. Esse tipo de prestação de serviços psicológicos deve estar ligado a práticas pontuais, informativas e focadas num determinado tema. Os psicólogos brasileiros podem legalmente realizar, por meio de TICs, ações de orientações psicológicas; de seleção de pessoal; de aplicação de testes; de supervisão do trabalho de psicólogos, realizada de forma eventual ou complementar ao processo de sua formação profissional presencial, além de atendimento eventual de clientes em trânsito e/ou que momentaneamente se encontrem impossibilitados de comparecer ao atendimento presencial (CFP, 2012). A escassez de estudos e normatizações técnicas e éticas sobre utilização de TICs por psicoterapeutas, no Brasil, somada a um crescimento significativo no uso dessas ferramentas justificam o investimento em pesquisas sobre o tema. Assim, o presente estudo objetivou investigar a percepção e o uso de TICs por psicoterapeutas em seus cotidianos pessoais e profissionais.

\section{Método}

\section{Delineamento e Cuidados Éticos}

Trata-se de uma pesquisa de delineamento quantitativo, exploratório, descritivo e de corte transversal. A presente pesquisa foi apresentada a um Comitê de Ética em Pesquisa e aprovada sob o protocolo de $\mathrm{n}^{\mathrm{o}} 579.827$.

\section{Amostra}

A amostra utilizada, selecionada por conveniência, foi de 155 psicoterapeutas gaúchos. Foram incluídos no estudo apenas psicoterapeutas graduados em Psicologia. Foram excluídos profissionais que não praticavam psicoterapia em seus consultórios particulares. Os psicólogos utilizados na amostra eram, em sua maioria, mulheres $(82,2 \%)$ e apresentavam idade entre 23 e 72 anos $(M=36,74 ; D P=11,45)$. Quanto ao tempo de exercício profissional, $44 \%$ da amostra possuía de 5 a 15 anos de prática clínica, 34\% apresentava até 5 anos de prática como psicoterapeutas e $22 \%$ possuía mais de 15 anos de experiência profissional. A abordagem teórica mais utilizada pela amostra em suas práticas clínicas é de orientação Psicanalítica (65\%), seguida da Terapia Cognitivo-comportamental (24\%). Também responderam à pesquisa psicoterapeutas de outras linhas teóricas como a Sistêmica, Gestalt-terapia, Psicodrama e de Orientação Integrativa, totalizando $11 \%$ da amostra. 


\section{Coleta de Dados}

Os participantes responderam um questionário online sobre percepção e uso de TICs. O questionário foi desenvolvido para a presente investigação e era composto de Termo de Consentimento Livre e Esclarecido; dados sociodemográficos e 34 perguntas sobre percepção, caracterização e frequência de uso de TICs. Para a construção desse instrumento foram consultados os dados do CGI (2012), do IBGE (2011), assim como dos estudos de Abreu et al. (2005) e de Leitão (2003). Realizou-se, previamente à coleta, um estudo piloto para aprimoramento do instrumento com 5 psicólogos clínicos que responderam ao questionário e deram sugestões de reformulações. Todas as sugestões foram consideradas pertinentes e acatadas. Tanto os participantes do estudo piloto quanto os participantes da amostra foram recrutados através de convites de participação de pesquisa, disponibilizados em sites de redes sociais e no site do Conselho Regional de Psicologia do Rio Grande do Sul CRP/07. Também foram enviados convites de participação de pesquisa, por email, para clínicos pertencentes a centros de formação de psicoterapeutas da grande Porto Alegre.

\section{Análise dos Dados}

Os dados coletados receberam tratamento estatístico descritivo e estatísticas inferenciais foram calculadas a fim de verificar as comparações entre os grupos de psicoterapeutas (sexo, abordagens teóricas, tempo de experiência clínica). Foi adotado o teste estatístico Chi-quadrado $\left(\chi^{2}\right)$ de Pearson, considerando o nível de significância $p<0,05$. Para esse tratamento estatístico foi utilizado o software SPSS, na versão 17.0.

\section{Resultados e Discussão}

\section{Uso de TICs por Psicoterapeutas}

Os resultados indicam que essas tecnologias estão significativamente presentes na vida pessoal dos psicoterapeutas pesquisados. A internet é acessada diariamente por $88 \%$ dos clínicos consultados e $9 \%$ declarou utilizar a rede, no mínimo, a cada 30 minutos. O aparelho mais utilizado para acessar a web é o telefone celular (55\%), seguido dos computadores portáteis, como os laptops, $(26 \%)$ e tablets (10\%). O computador de mesa é o aparelho menos utilizado para acesso à internet (9\%). O local de acesso mais frequente é a residência $(73,7 \%)$ e $59,4 \%$ dos psicoterapeutas pesquisados não possui wi- $f i$ no consultório. Ainda a internet é acessada com elevada frequência para realização de atividades relacionadas à comunicação (uso de sites de redes sociais, troca de emails), procura de informações em geral (uso de buscadores como o Google, consulta a jornais e revistas) e para pesquisas específicas sobre a profissão (busca de artigos científicos e de informações sobre encontros científicos). A realização de serviços financeiros é menos frequente em comparação a outros motivos para o acesso e a navegação da rede como fonte de lazer (jogos eletrônicos, assistir a filmes) se mostrou menos frequente (Figura 1).

Diferentemente dos achados qualitativos do começo dos anos 2000 (Leitão, 2003), os presentes resultados sugerem que os psicoterapeutas, atualmente, acessam a internet com maior frequência e regularidade do que há uma década atrás. Esse incremento pode ser reflexo do aumento expressivo de internautas no país. (CGI, 2012; IBGE, 2011). Somente no Rio Grande do Sul o percentual de acessos à rede mais que dobrou de 2005 a 2011. Estima-se que metade da população gaúcha é usuária da internet e que $66 \%$ dos internautas brasileiros a utilizam diariamente (CGI, 2012; IBGE, 2011). É interessante que $88 \%$ dos participantes do presente estudo façam uso diário da web, sugerindo que a proporção dos terapeutas que utiliza a web diariamente é maior que a proporção de pessoas em geral que utiliza a internet todos os dias. Os presentes resultados também refletem o aumento expressivo do número de computadores portáteis e redução dos computadores de mesa, além de uma tendência de crescimento de banda larga móvel no país (CGI, 2012). Esses resultados ainda confirmam os dados nacionais sobre local de acesso à internet. A residência é o lugar mais utilizado pelos brasileiros para navegação na web em detrimento do local de trabalho (CGI, 2012; IBGE, 2011). 


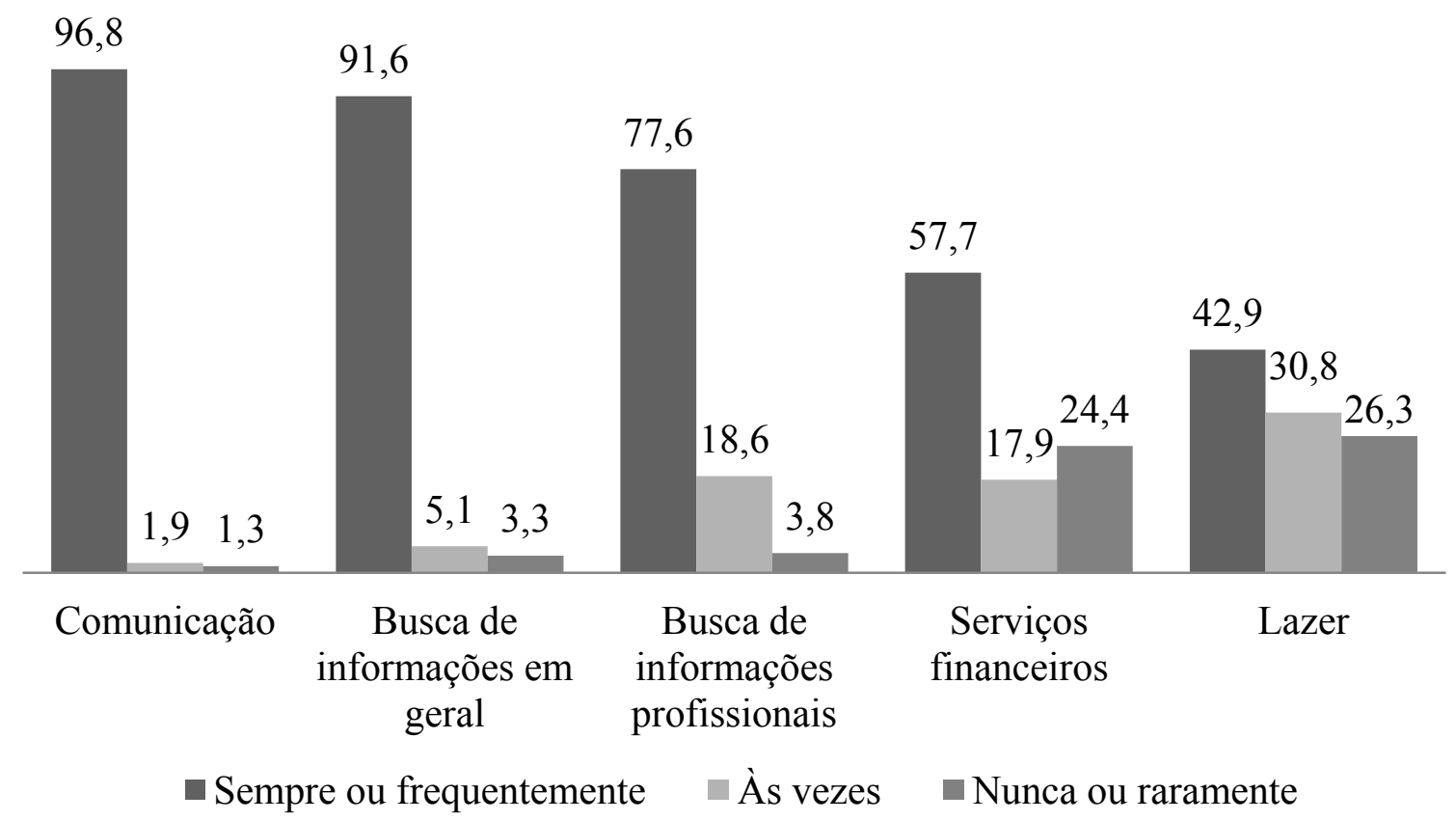

Figura 1. Frequência e propósito de uso da internet por psicoterapeutas.

Ainda os presentes resultados podem sugerir que as atividades realizadas na internet por psicoterapeutas continuam, como há uma década atrás, menos vinculadas a lazer e mais relacionadas à comunicação e pesquisas profissionais (Leitão, 2003). Ressalva-se, entretanto, que a comparação entre os achados atuais e os de Leitão (2003) deve ser relativizada tendo em vista as diferenças metodológicas entre os dois estudos.

Quanto ao uso da internet nas práticas profissionais, quase metade da amostra consultada $(42,9 \%)$ promove ou já promoveu seu trabalho clínico na web. Esse dado contrasta com os $20,7 \%$ de psicólogos norte-americanos que divulgam ou já divulgaram seus serviços pela internet (McMinn et al., 2011). Esse achado pode sugerir que o clínico gaúcho se mostra mais aberto a realizar esse tipo de atividade do que o americano. Ressalva-se, entretanto, que qualquer comparação entre os achados atuais e os norte-americanos deve ser relativizada tendo em vista as diferenças culturais entre as amostras e o período de coleta de dados dos dois estudos. Desse modo, alerta-se para o impacto das rápidas mudanças tecnológicas na produção dos resultados atuais. Assim, é possível que os psicoterapeutas do presente estudo se mostrem mais familiarizados com diversos tipos de TICs, como por exemplo com os sites de redes sociais, do que psicoterapeutas consultados há 4 anos atrás.

Também quanto ao uso da internet nas práticas profissionais, apenas $21,2 \%$ dos participantes afirmou prestar ou já ter prestado algum tipo de serviço psicológico via web. Essa baixa frequência parece similar aos dados americanos (McMinn et al., 2011) e também pode ser reflexo da atual legislação brasileira que atribui caráter exclusivamente experimental à prática da psicoterapia online (CFP, 2012). A baixa frequência na prestação de serviços online, encontrada no presente estudo, também pode indicar que esse é um tema polêmico e que divide as opiniões. Em uma pesquisa qualitativa com psicanalistas e psicoterapeutas sobre a possibilidade de prática clínica no ambiente da internet, foram encontradas divergências entre os entrevistados quanto à eficácia dessa modalidade de atendimento e se constatou uma visão prevalente de que a aliança terapêutica e vínculo terapêutico, processos indispensáveis na prática psicanalítica, só aconteceriam na presença física do paciente e clínico (Oliveira, 2009). Em outra pesquisa qualitativa, buscando compreender como psicoterapeutas avaliavam o oferecimento de psicoterapia pela internet, revelou que os profissionais pesquisa- 
dos, mesmo declarando um conhecimento insuficiente sobre o tema e mesmo sem terem contato com pessoas que tiveram a experiência de atendimento online, viam a prática com receio, preocupação, medo, preconceito, evitação e/ou como algo polêmico. Esses profissionais pesquisados também entendiam a falta do contato físico como uma desvantagem para a qualidade do vínculo e uma impossibilidade de que se mantivesse o papel do setting terapêutico (Pinhatti, Pieta, \& Gomes, 2011). Essas constatações contrastam com dados empíricos de uma pesquisa com pacientes que se submeteram à terapia via internet, na qual se observou a construção e manutenção da relação terapêutica semelhante às descritas pela literatura de psicoterapias presenciais (Meyer \& Prado, 2006).

Ainda no que diz respeito à utilização de TICs por psicoterapeutas, foram encontradas diferenças importantes entre a frequência de manejo dessas tecnologias no contexto pessoal e laboral da amostra. Esses resultados sugerem que, embora as TICs estejam significativamente presentes na vida pessoal dos clínicos, seu uso profissional é mais restrito. Essa restrição profissional se mostrou ainda mais significativa quando relacionada ao uso de sites de redes sociais. Esses achados corroboram o apontado na literatura sobre o tema (Ginory, Sabatier, \& Eth, 2012; McMinn et al., 2011). Verificou-se, por exemplo, que a troca de emails é uma atividade frequentemente realizada pelos psicoterapeutas consultados, mas poucos desses se comunicam com pacientes por essa via. O mesmo ocorreu com o uso do WhatsApp e com os sites de redes sociais (Figura 2). Apesar disso, não foram observadas diferenças significativas na frequência do uso de mensagens instantâneas via telefone celular (SMS/torpedo) na vida pessoal e profissional dos psicoterapeutas (Figura 3). Parece ser mais habitual para a amostra realizar esse tipo de comunicação com seus pacientes. $\mathrm{O}$ uso de videoconferência, através de programas como Skype, mostrou-se pouco usual tanto na vida pessoal quanto laboral dos clínicos consultados (Figura 3).

Esses achados são similares aos encontrados em pesquisa junto a psicólogos americanos (McMinn et al., 2011). Somente 5,5\% desses psicólogos clínicos declararam realizar serviços por email sempre ou frequentemente e apenas $12,5 \%$ se comunicava com pacientes para agendamento de sessões por essa via. A maioria $(99,07 \%) \mathrm{da}$ amostra americana jamais prestou serviços clínicos por meio de sites de redes sociais. Apenas $0,7 \%$ desses psicólogos declarou sempre ou fre-

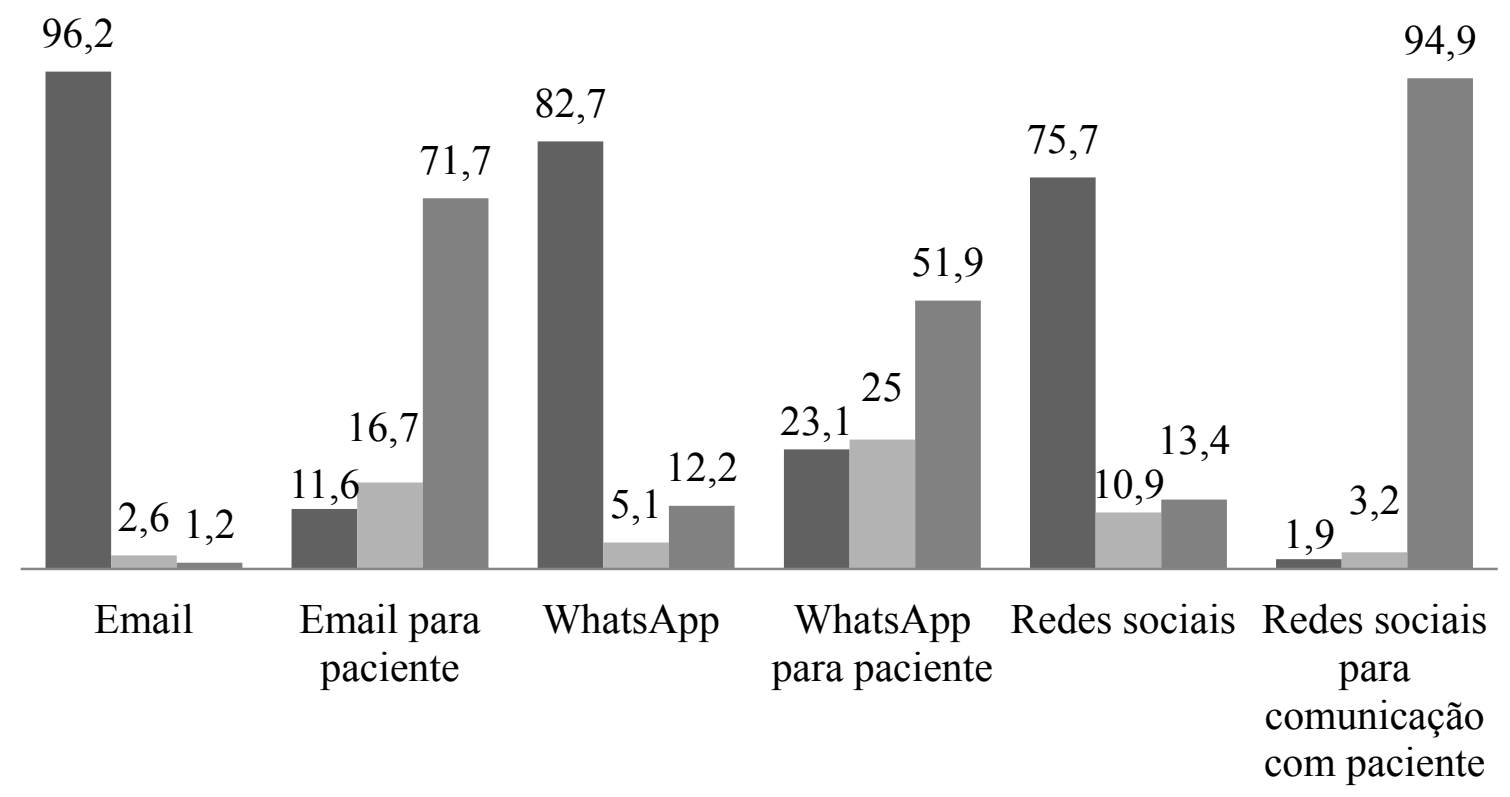

Sempre ou frequentemente $\square$ Às vezes $\quad$ Nunca ou raramente

Figura 2. Frequência de uso de Email, WhatsApp e redes sociais. 


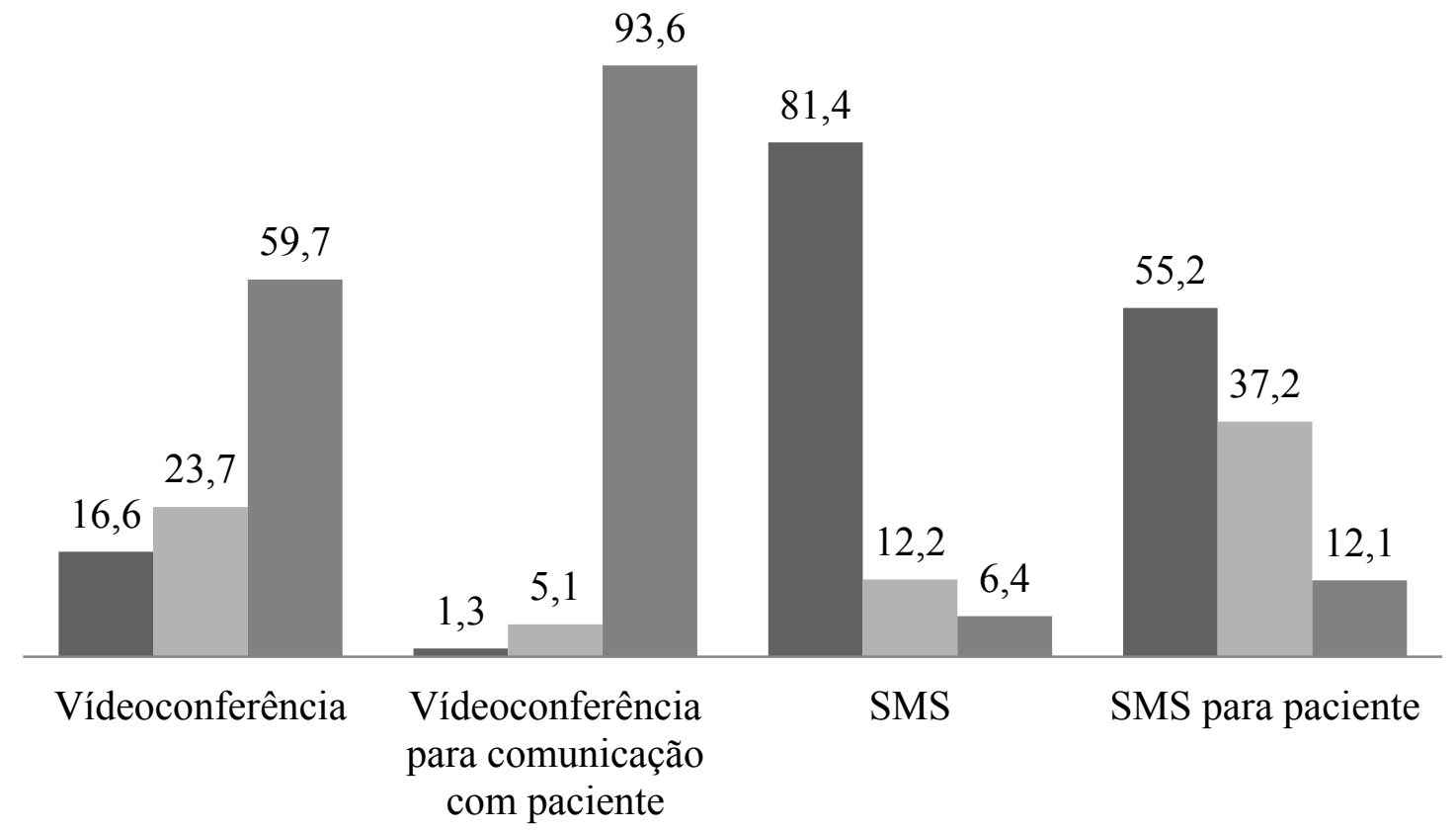

- Sempre ou frequentemente $\quad$ Às vezes $\quad$ Nunca ou raramente

Figura 3. Frequência de uso de videoconferência e SMS.

quentemente prestar serviços clínicos via Skype. Essas similaridades, entretanto, não se repetem no caso de comunicações por mensagens de texto do tipo SMS/torpedo com pacientes. Pouco mais da metade dos participantes do presente estudo $(55,2 \%)$ sempre ou frequentemente se comunica com seus pacientes ou clientes através dessa tecnologia contra $1,4 \%$ dos psicólogos americanos. Esse achado pode sugerir que o clínico gaúcho pode estar mais aberto a realizar esse tipo de atividade em relação ao americano. Novamente, essa comparação entre os achados atuais e os norte-americanos deve ser relativizada tendo em vista as diferenças culturais entre as amostras e o período de coleta de dados dos dois estudos.

Foram encontradas algumas diferenças significativas entre homens e mulheres e a frequência de uso de TICs no campo profissional. Os psicoterapeutas homens reportaram se comunicar através do email com seus atendidos mais frequentemente do que as mulheres $\left(X^{2}=\right.$ $9,565 ; p=0,048)$. Igualmente os homens reportaram prestar serviços psicológicos via internet com maior frequência do que elas $\left(X^{2}=8,296\right.$; $p=0,040)$. Esses achados tanto podem sugerir que os homens se mostram mais abertos a utili- zar as TICs em suas práticas clínicas, como também pode ser reflexo de indicadores estatísticos de que os homens são os maiores usuários da internet no país (CGI, 2012; IBGE, 2011).

Também foram encontradas diferenças significativas entre linha teórica e frequência de uso de TICs no campo profissional. Os psicoterapeutas de abordagem Cognitivo-comportamental reportaram se comunicar com seus atendidos através do email com maior frequência do que terapeutas de outras abordagens $\left(X^{2}=226,638\right.$; $p<0,001)$. Igualmente os psicoterapeutas de orientação Cognitivo-comportamental se comunicam mais frequentemente com seus pacientes/ clientes através do WhatsApp $\left(X^{2}=173,365\right.$; $p<0,001)$, do Skype $\left(X^{2}=179,106 ; p<0,001\right)$, de mensagem de texto do tipo SMS/torpedo $\left(X^{2}\right.$ $=169,543 ; p<0,001)$ e via sites de redes sociais $\left(X^{2}=197,172 ; p<0,001\right)$. Esses terapeutas ainda prestam $\left(X^{2}=180,998 ; p<0,001\right)$ e divulgam $\left(X^{2}\right.$ $=187,685 ; p<0,001)$ serviços psicológicos, pela internet, com mais frequência que os psicanalistas e clínicos de outras linhas teóricas.

Esses achados podem sugerir que, embora o uso profissional de TICs pelos psicoterapeutas pesquisados seja mais restrito do que o uso 
pessoal, em clínicos de abordagem Cognitivo-comportamental essa restrição é menor. No presente estudo, esses terapeutas se mostraram mais abertos a utilizar as TICs em suas práticas clínicas do que os psicanalistas e terapeutas de outras linhas teóricas. É possível que esses resultados se devam às características mais diretivas e focais presentes nas técnicas e teorias da abordagem Cognitivo-comportamental (Beck, 2000).

Ainda foram encontradas diferenças estatisticamente significativas entre tempo de experiência clínica e frequência de uso de TICs no campo profissional. Os psicoterapeutas com até cinco anos de experiência psicoterápica, ou seja, com menos tempo de prática clínica reportaram se comunicar com seus atendidos através do email com maior frequência que os demais $\left(X^{2}\right.$ $=177,798 ; p<0,001)$. Os psicoterapeutas menos experientes também divulgam mais seus serviços profissionais na internet em relação aos demais $\left(X^{2}=169,020 ; p<0,001\right)$. Já os participantes com mais de 15 anos de prática clínica, ou seja, com mais tempo de experiência profissional se comunicam com seus atendidos através do WhatsApp $\left(X^{2}=168,316 ; p<0,001\right)$, do torpedo $\left(X^{2}=\right.$ $168,185 ; p<0,001)$ e via sites de redes sociais $\left(X^{2}\right.$ $=164,715 ; p<0,001)$ com maior frequência que os demais. Os participantes que possuíam entre cinco a 15 anos de prática clínica reportaram se comunicar com seus atendidos através do Skype $\left(X^{2}=177,798 ; p<0,001\right)$ com maior frequência que os demais e também são o grupo que presta serviços psicológicos via internet com maior frequência $\left(X^{2}=177,798 ; p<0,001\right)$. Apesar do uso profissional de TICs pelos participantes ser mais restrito do que o uso pessoal, os resultados indicam que os psicólogos mais experientes e provavelmente mais velhos fazem uso mais frequente de um maior número de ferramentas tecnológicas de em seus cotidianos profissionais do que os terapeutas menos experientes. Esses resultados vão de encontro a uma expectativa de que psicólogos mais jovens estariam potencialmente mais familiarizados com novas tecnologias e que por isso utilizariam as TICs em suas práticas clínicas com maior frequência. Essa expectativa foi confirmada em dados norte-americanos que apon- taram diferenças estatisticamente significativas entre o tempo de experiência clínica e frequência no uso de tecnologias, sugerindo que psicólogos mais jovens fariam mais uso de TICs do que os mais experientes (McMinn et al., 2011). Ressalva-se, entretanto, que os resultados do presente estudo devem ser discutidos tendo em vista que os clínicos mais experientes provavelmente possuem uma clientela maior em comparação a psicoterapeutas recém-formados, o que potencialmente aumentaria a frequência de contato com clientes via TICs. É ainda possível que o tempo de exercício clínico favoreça um uso profissional de TICs mais frequente. É provável que psicoterapeutas mais experientes se sintam tecnicamente e eticamente mais seguros, capazes e confortáveis em realizar comunicações com seus atendidos via TICs do que seus colegas menos experientes.

\section{Percepção sobre TICs por Psicoterapeutas}

A maioria dos participantes do presente estudo $(94,9 \%)$ acredita que as TICs estão cada vez mais presentes na vida de seus atendidos. Não foram encontradas diferenças estatísticas significativas quanto ao sexo $\left(X^{2}=2,062\right.$; $p=0,560)$, linha teórica $\left(X^{2}=2,738 ; p=0,841\right)$ e tempo de experiência profissional $\left(X^{2}=6,848\right.$; $p=0,335)$, indicando que para todos os grupos de psicoterapeutas consultados essa percepção é similar. Ainda 78,2\% dos participantes percebe que é cada vez mais frequente ser contatado por pacientes via TICs. Essa percepção é a mesma, independentemente do sexo $\left(X^{2}=4,293\right.$; $p=0,368)$, linha teórica $\left(X^{2}=7,266 ; p=0,508\right)$ e tempo de experiência profissional $\left(X^{2}=10,993\right.$; $p=0,202)$ dos participantes. Esses achados podem ser reflexo dos dados estatísticos de aumento expressivo do consumo de TICs no país (CGI, 2012; IBGE, 2011). Apesar disso, é interessante que somente pouco mais da metade dos clínicos consultados $(54,4 \%)$ tem procurado estudar sobre o impacto dessas novas tecnologias no campo das psicoterapias. Nos achados qualitativos com 16 psicoterapeutas brasileiros sobre uso de internet do começo dos anos 2000, poucos clínicos referiram procurar informações sobre os 
avanços teóricos e empíricos no campo da web e psicologia (Leitão, 2003). Parece que, como há uma década atrás, os psicoterapeutas resistem em acessar informações sobre a presença, atravessamentos e avanços tecnológicos na área da psicologia clínica.

Ainda no que diz respeito à percepção sobre TICs, $69,9 \%$ dos participantes referiu fazer uso comedido dessas ferramentas devido a sua profissão. Esses resultados corroboram os já citados dados qualitativos sobre uso restrito da internet por psicoterapeutas (Abreu et al., 2005; Leitão, 2003), além de serem similares aos achados sobre manejo reduzido de tecnologias por psicólogos americanos em suas práticas clínicas (McMinn et al., 2011). No presente estudo foi constatada diferença significativa quanto ao sexo do psicoterapeuta e uso comedido de TICs, apontando que as mulheres manejam essas ferramentas tecnológicas com maior restrição do que os homens $\left(X^{2}=11,623 ; p=0,020\right)$. Esse resultado, aliado aos já citados achados de que os homens se comunicam mais com seus atendidos via email e prestam serviços psicológicos via internet com maior frequência que as mulheres, reforçam a inferência de que psicoterapeutas homens se mostram mais abertos a utilizar as TICs em suas práticas clínicas. Esse achado é notável tendo em vista que a psicologia clínica é majori- tariamente exercida por mulheres no Brasil (Godim, Bastos, \& Peixoto, 2010).

Embora, no presente estudo, 69,9\% dos participantes acredite fazer uso comedido de TICs em razão de sua profissão, à exceção do recebimento de solicitação de amizades de pacientes pelo Facebook, não se observou um significativo desconforto nos participantes ao se comunicarem com seus pacientes/clientes através de outros tipos de TICs. Quando questionados sobre uma possível sensação de desconforto ao receberem email, torpedo ou mensagem via WhatsApp de seus pacientes/clientes, a maioria dos clínicos consultados pareceu não se incomodar em estabelecer esse tipo de comunicação (Figura 4). Não foram identificadas diferenças significativas quanto ao desconforto na comunicação com pacientes através de TICs em relação ao sexo $\left(X^{2}\right.$ $=3,873 ; p=0,423)$ e tempo de experiência $\left(X^{2}=\right.$ $12,830 ; p<0,118)$ profissional. Infere-se, assim, que, independente do sexo do psicoterapeuta ou tempo de experiência profissional, a maioria dos clínicos consultados não se sente desconfortável ao se comunicar com atendidos via email, torpedo ou WhatsApp. Do mesmo modo, independente do sexo ou tempo de prática clínica, a metade dos profissionais consultados refere desconforto com o recebimento de solicitações de amizade de pacientes/clientes via Facebook.

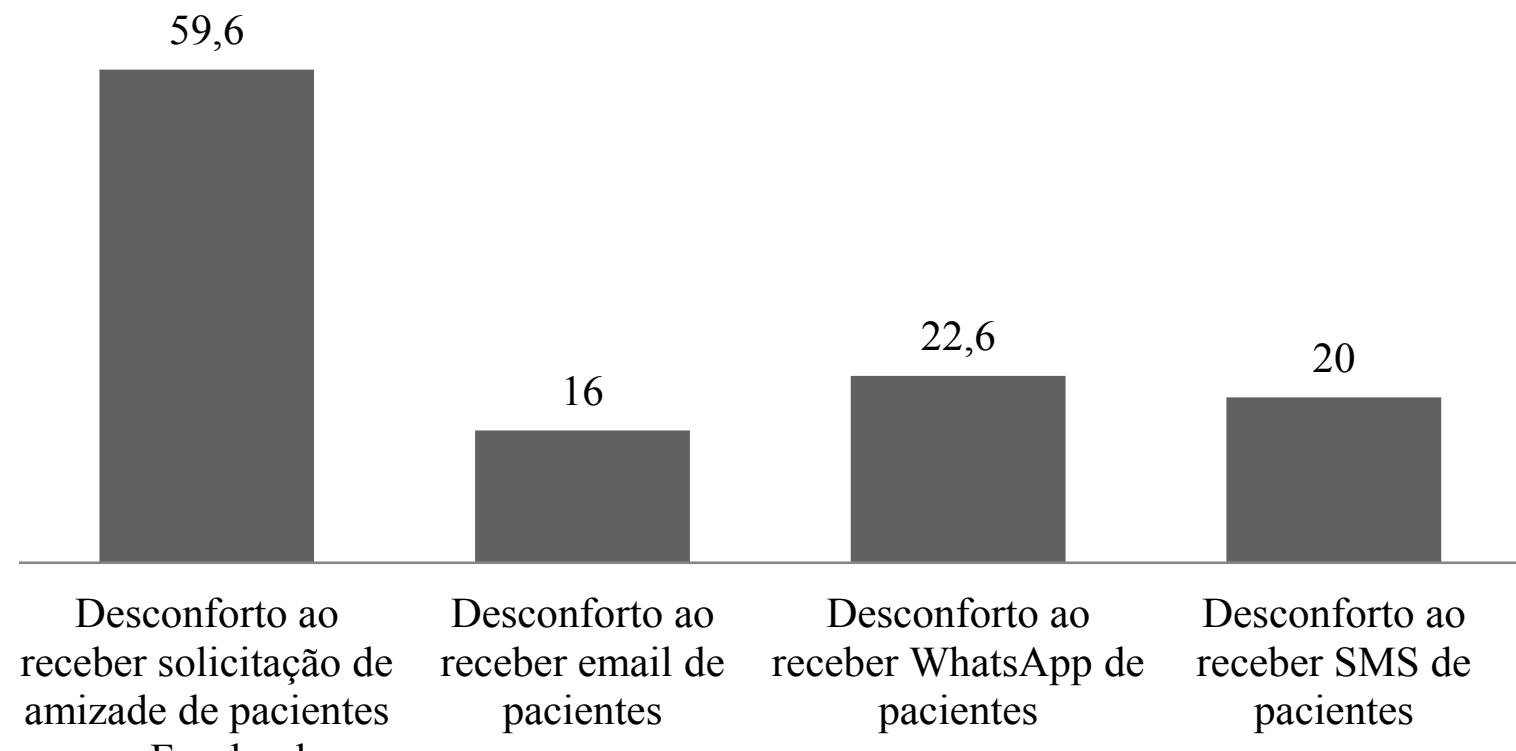

no Facebook

Figura 4. Frequência de percepção de desconforto ao se comunicar com pacientes via TICs. 
A percepção de desconforto se mostrou diferente dependendo da abordagem teórica adotada pelos participantes desse estudo. O grupo de psicanalistas reportou maior desconforto com o recebimento de emails de pacientes em comparação aos demais $\left(X^{2}=25,190 ; p<0,001\right)$. Em contrapartida, o grupo de clínicos de abordagem Cognitivo-Comportamental referiu com menor frequência sentimento de desconforto com o recebimento desses emails $\left(X^{2}=25,190\right.$; $p<0,001)$. Igualmente o grupo de psicanalistas reportou maior desconforto com o recebimento de solicitações de amizade, via Facebook, de pacientes $\left(X^{2}=32,187 ; p<0,001\right)$ e clínicos de abordagem Cognitivo-Comportamental referiram se sentirem menos desconfortáveis com o recebimento dessas solicitações $\left(X^{2}=32,187\right.$; $p<0,001)$. O grupo de psicoterapeutas de outras abordagem teóricas, que não a Psicanalítica e Cognitivo-comportamental, mostraram-se mais desconfortáveis ao receberem mensagem de pacientes pelo WhatsApp $\left(X^{2}=18,132 ; p=0,020\right)$ e os psicólogos de orientação Cognitivo-Comportamental reportaram menos desconforto com o recebimento desse tipo de mensagem $\left(X^{2}=\right.$ $18,132 ; p=0,020)$. Esses resultados estão em consonância com os achados de que os psicólogos de orientação Cognitivo-Comportamental divulgam e prestam serviços clínicos pela internet com maior frequência e também mais frequentemente se comunicam com atendidos via email, WhatsApp, Skype, torpedo e através de sites de redes sociais. Reforça-se, assim, a inferência de que, embora o manejo profissional de TICs seja mais restrito do que o uso pessoal, em clínicos de orientação Cognitivo-Comportamental a restrição parece ser menor. Esses terapeutas são o grupo de profissionais que se sentem mais confortáveis e se mostram mais abertos a utilizar as TICs em suas práticas clínicas. Em contrapartida, os psicólogos de orientação Psicanalítica parecem reportar maior desconforto com a utilização profissional de TICs. Esse achado ganha importância tendo em vista que a linha teórica Psicodinâmica fundamenta o trabalho da maioria dos psicólogos clínicos em atividade no país (Godim et al., 2010). É possível que esse achado se deva às características menos focais e dire- tivas das abordagens psicanalíticas (Zimerman, 1999). De igual modo, a ênfase na valorização da preservação do setting psicoterapêutico para manejo de aspectos transferenciais e contratansferencias, própria das técnicas e teorias psicodinâmicas, pode contribuir para o quadro de maior desconforto na a utilização profissional de TICs por esses clínicos (Eizirik, Aguiar \& Schestatsky, 2015).

\section{Considerações Finais}

Sobre o uso de TICs por psicoterapeutas, os resultados indicam que essas tecnologias estão significativamente presentes na vida pessoal dos psicoterapeutas pesquisados, entretanto, seu uso profissional é mais restrito. Esse uso profissional restrito se mostrou ainda mais significativo quando relacionado aos sites de redes sociais. Os psicoterapeutas, atualmente, acessam a internet, com elevada frequência e regularidade, para diversos fins, acompanhando o recente crescimento no número de internautas no país. A internet é mais utilizada pelos psicoterapeutas para fins de comunicação e menos acessada para propósitos de lazer. Alguns resultados sugerem que os homens se mostram mais abertos a utilizar as TICs em suas práticas clínicas do que as mulheres, assim como os clínicos de orientação Cognitivo-comportamental e os psicoterapeutas com mais tempo de experiência profissional. Alguns resultados também sugerem que os clínicos da presente amostra utilizam com menor restrição as TICs, no contexto profissional, do que os clínicos norte-americanos. Esses resultados podem ser explicados por diferenças culturais e pelo fato dos achados da presente amostra serem mais atualizados do que os dados americanos. Quanto à percepção sobre TICs, independente do gênero, linha teórica e tempo de prática profissional, a maioria dos psicoterapeutas acredita que essas novas tecnologias estão cada vez mais presentes na vida de seus atendidos. Entretanto, menos da metade dos clínicos tem procurado estudar sobre o impacto dessas novas tecnologias no campo das psicoterapias. À exceção do recebimento de solicitação de amizades de pacientes, via Facebook, os clínicos pesquisados não se sentem 
desconfortáveis na comunicação com pacientes através de TICs, com destaque ao grupo dos profissionais de orientação Cognitivo-comportamental, que se sentem mais confortáveis com a utilização de todos os tipos de tecnologias, inclusive os sites de redes sociais.

Ponderando-se sobre as implicações práticas da presente pesquisa é importante registrar que a crescente entrada das TICs no cotidiano dos brasileiros, aliada às lacunas teóricas e legais sobre essa temática, justificam estudos que visem compreender como psicoterapeutas têm percebido e lidado com a presença das TICs na clínica psicoterápica. A presente pesquisa apontou para a necessidade de investimentos em estudos empíricos sobre TICs, especialmente sobre o uso de redes sociais por psicoterapeutas, pois foi a tecnologia com maior restrição de uso e que parece gerar mais desconforto nos profissionais. Parece que o manejo profissional adequado de TICs deveria cada vez mais constar no currículo de graduação em Psicologia e no de centros de formação técnica de psicoterapeutas. Os resultados da presente pesquisa ainda alertam para a necessidade de investimentos na elaboração de resoluções e orientações mais específicas sobre como psicoterapeutas devem conduzir seus contatos com atendidos via TICs. Registra-se como limitação da presente pesquisa a ausência de análises estatísticas sobre a diferença de proporção entre número de psicanalistas e número de terapeutas de outras abordagens. Outra limitação refere-se ao uso de amostra por conveniência e não randomizada, o que diminui o poder de generalização dos achados aqui descritos. Além disso, os resultados da presente pesquisa devem ser pensados dentro do contexto da amostra utilizada, tendo em vista que o uso de TICs, no país, não está igualmente distribuído e apresenta variações sociodemográficas, econômicas e regionais. Sugere-se o desenvolvimento de estudos similares, com amostra nacional e randomizada, para descrição mais generalizada do uso de TICs por psicoterapeutas. Sugere-se também o desenvolvimento de estudos empíricos de delineamento qualitativo para aprofundamento da compreensão desse tema.

\section{Referências}

Abreu, R. S., Leitão, C. F., \& Nicolaci-da-Costa, A. M. (2005). Profissionais à deriva: Professores e psicoterapeutas na sociedade em rede. Interações, 19(10), 151-174. Recuperado em http:// www.redalyc.org/articulo.oa? $\mathrm{id}=35401908$

Barnett, J. E. (2011). Utilizing technological innovations to enhance psychotherapy supervision, training, and outcomes. Psychotherapy, 48(2), 103-108. doi:10.1037/a0023381

Beck, A. (2000). O poder integrador da terapia cognitiva (M. Monteiro, Trad.). Porto Alegre, RS: Artes Médicas Sul.

Carvalho, M. S. R. M. (2006). A trajetória da Internet no Brasil: Do surgimento das redes de computadores à instituição dos mecanismos de governança (Dissertação de mestrado, Universidade Federal do Rio de Janeiro, RJ, Brasil).

Comitê Gestor da Internet no Brasil. (2012). Pesquisa sobre o uso das tecnologias de informação e comunicação no Brasil: TIC Domicílios e TIC Empresas 2011. Recuperado em http:// op.ceptro.br/cgi-bin/cetic/tic-domicilios-e-empresas-2011.pdf

Conselho Federal de Medicina. (2011). Resolução $n^{\circ}$ 1.974/2011. Recuperado em http:// www.portalmedico.org.br/resolucoes/ CFM/2011/1974 2011.htm

Conselho Federal de Psicologia. (2000). Resolução do Conselho Federal de Psicologia $N^{\circ}$ 003/2000. Recuperado em http://www.crprs.org.br/upload/ legislacao/legislacao40.pdf

Conselho Federal de Psicologia. (2005). Código de Ética Profissional do Psicólogo. Recuperado em http://site.cfp.org.br/wp-content/uploads/2012/07/codigo_etica.pdf

Conselho Federal de Psicologia. (2012). Resolução $C P F \mathrm{~N}^{\circ}$ 011/ 2012. Recuperado em http://site. cfp.org.br/wp-content/uploads/2012/07/Resoluxo_CFP_nx_011-12.pdf

Eizirik, C. L., Aguiar, R., \& Schestatsky, S. S. (2015). Psicoterapia de Orientação Analítica: Fundamentos teóricos e clínicos. Porto Alegre, RS: ArtMed

Godim, S. M. G., Bastos, A. V. B., \& Peixoto, L S. A. (2010). Áreas de atuação, atividades e abordagens teóricas do psicólogo brasileiro. In A. V. B. Bastos \& S. M. Godem (Eds.), $O$ 
trabalho do psicólogo no Brasil: Um exame à luz das categorias da psicologia organizacional e do trabalho (pp. 174-199). Porto Alegre, RS: ArtMed

Ginory, A., Sabatier, L. M., \& Eth, S. (2012). Addressing therapeutic boundaries in social networking. Psychiatry, 75(1), 40-48. doi:10.1521/ psyc.2012.75.1.40

Instituto Brasileiro de Geografia e Estatística. (2011). Pesquisa Nacional por Amostra de Domicilios: Acesso à internet e posse de telefone móvel celular para uso pessoal - PNAD. Recuperado em $\mathrm{ftp} / / / \mathrm{ftp}$. ibge.gov.br/Acesso_a_internet_e_posse_celular/2011/PNAD_Inter_2011.pdf

Kolmes, K. (2012). Social media in the future of professional psychology. Professional Psychology: Research and Practice, 43(6), 606-612. doi:10.1037/a0028678

Leitão, C. F (2003). Os impactos subjetivos da Internet: Reflexões teóricas e clínicas (Tese de doutorado, Pontifícia Universidade Católica do Rio de Janeiro, RJ, Brasil).

Leitão, C. F., \& Nicolaci-da-Costa, A. M. (2001). Psicologia clínica e informática: Por que essa inusitada aproximação? Psicologia Clínica, 12(2), 189-205. Recuperado em http://www.maxwell. vrac.puc-rio.br/4122/4122_4.PDF

Levisky, R. B., \& Silva, M. C. R. (2010). A invasão das novas formas de comunicação no setting terapêutico. Vínculo - Revista do NESME, 7(1), 63-70. Recuperado em http://pepsic.bvsalud. org/scielo.php?script=sci

Meyer, S. B., \& Prado, O. Z. (2006) Avaliação da relação assimétrica na terapia assíncrona via internet. Psicologia em Estudo, 11(2), 247-257. Recuperado em http://www. scielo.br/scielo.php?script=sci_arttext\&pid $=$ S1413-73722006000200003

McMinn, M. R., Bearse, J., Heyne L. K., Smithberger, A., \& Erb, A. L. (2011). Technology and independent practice: Survey findings and implications. Professional Psychology: Research and Practice, 42(2), 176-184. doi:10.1037/a0022719
Oliveira, P. C. S. (2009). O divã virtual e a linguagem do atendimento psicanalitico on-line no ciberespaço (Dissertação de mestrado, Universidade Estadual do Norte Fluminense Darcy Ribeiro, Campos dos Goitacazes, RJ, Brasil).

Pinhatti, M. M., Pieta, M. A. M., \& Gomes, W. B. (2011). Terapia pela internet: Limites e possibilidades na percepção de psicoterapeutas [Resumo e pôster]. Trabalho apresentado no XXIII Salão de Iniciação Científica da Universidade Federal do Rio Grande do Sul, Porto Alegre, RS, Brasil.

Sfoggia, A., Kowacs, C., Gastaud, M. B., Laskoski, P. B., Bassols, A. M., Severo, C. T., ...Eizirik, C. L. (2014). Therapeutic relationship in the web: To face or not to face? Trends Psychiatry Psychotherapy, 36(1), 3-10. doi:10.1590/22376089-2013-0048

Silverman, W. H. (2013). The future of psychotherapy: One editor's perspective. Psychotherapy, 50(4), 484-489. doi:10.1037/a0030573

Veloso, R. (2011). Tecnologias da informação e comunicação: Desafios e perspectivas. São Paulo, SP: Saraiva.

Zimerman, D. E. (1999). Fundamentos psicanalíticos: Teoria, técnica, clínica - Uma abordagem didática. Porto Alegre, RS; ArtMed. 